\title{
Seven years' experience with alendronate in postmenopausal Japanese women with osteoporosis
}

This article was published in the following Dove Press journal:

Therapeutics and Clinical Risk Management

16 April 2010

Number of times this article has been viewed

\author{
Jun Iwamoto' \\ Yoshihiro Sato ${ }^{2}$ \\ Mitsuyoshi Uzawa ${ }^{3}$ \\ Tsuyoshi Takeda' \\ Hideo Matsumoto' \\ 'Institute for Integrated Sports \\ Medicine, Keio University School \\ of Medicine, Tokyo, Japan; \\ ${ }^{2}$ Department of Neurology, \\ Mitate Hospital, Fukuoka, Japan; \\ ${ }^{3}$ Department of Orthopaedic \\ Surgery, Keiyu Orthopaedic Hospital, \\ Gunma, Japan
}

\begin{abstract}
A retrospective study was performed to evaluate the outcome of alendronate (ALN) treatment for seven years in postmenopausal Japanese women with osteoporosis. Forty-seven postmenopausal women with osteoporosis (mean age at baseline 65.7 years) treated with ALN for over seven years in our outpatient clinic were analyzed. Lumbar spine bone mineral density (BMD) was measured using dual energy X-ray absorptiometry, and urinary levels of cross-linked N-terminal telopeptides of type I collagen (NTX) and serum alkaline phosphatase (ALP) were monitored during the seven-year treatment period. Urinary NTX and serum ALP levels decreased $(-48.2 \%$ at three months and $-15.7 \%$ at seven years, respectively) and lumbar spine BMD increased $(+12.8 \%$ at seven years $)$ compared with baseline values. No serious adverse events were observed, including osteonecrosis of jaw, atypical femoral diaphysis fractures, or atrial fibrillation. To our knowledge, this is the first report of the outcome of ALN treatment for seven years in Japanese patients with osteoporosis. ALN successfully suppressed bone turnover and increased lumbar spine BMD from the baseline value over the course of the seven-year treatment period without causing any severe adverse events.
\end{abstract}

Keywords: alendronate, osteoporosis, long-term treatment, bone mineral density, bone turnover

\section{Background}

Osteoporosis most commonly affects postmenopausal women, placing them at increased risk of fractures. Alendronate (ALN) is widely used for the treatment of postmenopausal osteoporosis. The Fracture Intervention Trial demonstrated the antifracture efficacy of ALN for vertebral, nonvertebral, hip, and wrist fractures in postmenopausal women with osteoporosis. ${ }^{1,2}$ A recent systematic review analyzing 11 randomized controlled trials including 12,068 women has confirmed both clinically important and statistically significant reductions in vertebral, nonvertebral, hip, and wrist fractures for secondary prevention of osteoporosis (gold standard evidence). ${ }^{3}$ ALN is regarded as a first-line drug for the treatment of osteoporosis in Japan.

The long-term efficacy of antifracture drugs needs to be established. Bone et $\mathrm{al}^{4}$ reported their experience using ALN to treat postmenopausal Western women with osteoporosis for a period of 10 years. Ten years of ALN treatment produced an increase in bone mineral density (BMD) of $13.7 \%$ at the lumbar spine, $10.3 \%$ at the trochanter, $5.4 \%$ at the femoral neck, and $6.7 \%$ at the total proximal femur, compared with baseline values. Safety data, including fractures and stature, did not suggest that prolonged treatment resulted in any loss of benefit. Thus, the therapeutic effects of ALN were sustained, and the drug was well tolerated over a 10-year period.
Institute for Integrated Sports Medicine,

35 Shinanomachi, Shinjuku-ku,

Tokyo 160-8582, Japan

Tel +8I 33353 I 2 I I

Fax +8I 333529467

Email jiwamoto@sc.itc.keio.ac.jp 
In Japan, results of randomized controlled trials testing the effects of short-term (1-3 years) ALN treatment on $\mathrm{BMD}$, bone turnover, and fracture incidence have been reported in postmenopausal women with osteoporosis..$^{5-8}$ ALN reduces bone turnover, increases lumbar and hip BMD, and prevents vertebral fractures. Recently, we reported that ALN successfully reduced bone turnover and maintained metacarpal BMD for five years in older postmenopausal Japanese women with osteoporosis or osteopenia and clinical risk factors for fractures. ${ }^{9}$ To our knowledge, however, the outcome of long-term (more than five years) ALN treatment has not been previously reported in Japanese patients. Therefore, a retrospective study was performed to evaluate the outcome of ALN treatment for seven years in postmenopausal Japanese women with osteoporosis. The primary endpoint was lumbar spine $\mathrm{BMD}$, and the secondary endpoint was biochemical markers. Adverse events such as osteonecrosis of the jaw (ONJ), atypical femoral diaphysis fractures, and atrial fibrillation, ${ }^{10-12}$ as well as incident osteoporotic fractures were also assessed.

\section{Subjects and methods Subjects}

Forty-seven postmenopausal Japanese women (age range 52-83 years, mean age 65.7 years at the beginning of treatment) who had been treated with ALN for over seven years were recruited from the outpatient clinic at Keiyu Orthopaedic Hospital (Gunma, Japan) during the period between July 1 and December 30, 2009. Exclusion criteria were a history of reflux esophagitis, gastric or duodenal ulcer, gastrectomy, or bone diseases secondary to primary hyperparathyroidism, hyperthyroidism, Cushing's syndrome, multiple myeloma, rheumatoid arthritis, and osteogenesis imperfecta.

All the patients had been diagnosed as having osteoporosis according to the Japanese diagnostic criteria. ${ }^{13,14}$ Namely, patients with a BMD $<70 \%$ of the young adult mean (YAM) or a BMD $70 \%-80 \%$ of the YAM along with a history of osteoporotic fractures were diagnosed as having osteoporosis. Preliminary screening included a medical history, physical examination, plain $\mathrm{X}$-rays of the thoracic and lumbar spine, lumbar spine BMD measurement, and blood and urinary biochemical tests, including serum calcium, phosphorus, alkaline phosphatase (ALP) and urinary cross-linked N-terminal telopeptides of type I collagen (NTX). Lumbar spine BMD was used for the diagnosis of osteoporosis.
The patients had been treated with ALN (5 mg daily or $35 \mathrm{mg}$ weekly) over seven years. The doses indicated in parentheses above are the doses used in Japan for the treatment of postmenopausal women with osteoporosis and have been recognized as being safe and effective. ${ }^{7}$ Daily ALN was available throughout the study period, but weekly ALN only became available in October 2006. All the patients had been treated with daily ALN and then switched to weekly ALN just after October 2006. The effects of daily and weekly ALN on BMD and bone turnover markers, as well as incidence of side effects, were reported to be similar in postmenopausal Japanese women with osteoporosis. ${ }^{8}$ The subjects did not receive any elementary calcium or natural vitamin D supplementation.

Urinary levels of NTX were measured three months after the start of treatment, based on the results of our previous study. ${ }^{15}$ Serum levels of calcium, phosphorus, and ALP, and lumbar spine BMD were measured every year after the start of treatment. Serum bone-specific ALP levels were also measured at seven years after the start of treatment. The outcome of seven years of ALN treatment was evaluated. The present study was approved by the Ethics Committee of Keiyu Orthopaedic Hospital.

\section{Assessment of vertebral fractures}

Plain lateral X-ray films of the thoracic and lumbar spine were obtained at baseline to detect evidence of morphometric vertebral fractures. According to the Japanese criteria, a vertebral fracture was defined according to the vertebral height on lateral X-ray films. ${ }^{13,14}$ Briefly, the vertebral height was measured at the anterior (A), central (C), and posterior $(\mathrm{P})$ aspects of the vertebral body, and the presence of a vertebral fracture was confirmed when a reduction in vertebral height of more than $20 \%$ (A, C, and P) compared with the height of the adjacent vertebrae was observed, the $\mathrm{C} / \mathrm{A}$ or $\mathrm{C} / \mathrm{P}$ was less than 0.8 , or the $\mathrm{A} / \mathrm{P}$ was less than 0.5 . The assessment for vertebral fractures was performed at the T4-L4 level.

\section{Serum ALP, calcium, phosphorus, bone-specific ALP, and urinary NTX}

Serum calcium, phosphorus, and ALP levels were measured by standard laboratory techniques (normal range $8.4-10.2 \mathrm{mg} / \mathrm{dL}$, 2.5-4.5 mg/dL, and 135-310 IU/L, respectively). Serum bone-specific ALP levels were measured by enzyme immunoassay (EIA, normal range for Japanese women 7.9-29.0 U/L). ${ }^{16}$ Urinary NTX levels were measured by enzyme-linked immunosorbent assay (ELISA, normal range 9.3-54.3 $\mathrm{nM}$ bone collagen equivalent $[\mathrm{BCE}] / \mathrm{mM} \mathrm{Cr}){ }^{16}$ 


\section{Measurement of lumbar spine BMD}

BMD of the lumbar spine (L1-L4) in the anteroposterior (AP) view was measured using dual-energy $\mathrm{X}$-ray absorptiometry (DXA) with Hologic QDR 1500W apparatus (Bedford, MA). The coefficient of variation $(100 \times$ standard deviation/mean $)$ of five measurements, with repositioning within 72 hours each time, was less than $1.2 \%$ in three persons.

\section{Statistical analysis}

Data were expressed as the mean \pm standard deviation (SD) in the table and the mean $\pm 95 \%$ confidence interval (CI) for calcium, phosphorus, and ALP, and the median $\pm 95 \%$ CI for urinary NTX in the figures. The significance of longitudinal changes in the BMD and biochemical markers was determined using a one-way analysis of variance (ANOVA) with repeated measurements. All statistical analyses were performed using the Stat View-J5.0 program on a Windows computer. A significance level of $P<0.05$ was used for all the comparisons.

\section{Results}

\section{Characteristics of subjects at start of treatment}

Table 1 shows the baseline characteristics of the study subjects at the start of ALN treatment. The mean age was 65.7 years. The mean lumbar spine BMD was $0.638 \mathrm{~g} / \mathrm{cm}^{2}(62.7 \%$ of YAM). Twenty-three subjects (48.9\%) had prevalent vertebral fractures, and the mean number of prevalent vertebral fractures per subject was 2.5 . The mean level of urinary

Table I Characteristics of study subjects

\begin{tabular}{lll}
\hline & Mean \pm SD & Range \\
\hline Age (years) & $65.7 \pm 8.6$ & $52-83$ \\
Height $(\mathrm{m})$ & $1.48 \pm 0.07$ & $1.35-1.62$ \\
Body weight $(\mathrm{kg})$ & $45.4 \pm 6.6$ & $32-59$ \\
Body mass index $\left(\mathrm{kg} / \mathrm{m}^{2}\right)$ & $20.5 \pm \mathrm{I} .1$ & $15.6-25.9$ \\
Lumbar spine BMD $\left(\mathrm{g} / \mathrm{cm}^{2}\right)$ & $0.638 \pm 0.09 \mathrm{I}$ & $0.433-0.788$ \\
\%YAM of lumbar spine BMD (\%) & $62.7 \pm 9.0$ & $42.6-77.4$ \\
Serum calcium (mg/dL) & $9.4 \pm 0.4$ & $8.6-0.3$ \\
Serum phosphorus (mg/dL) & $3.3 \pm 0.5$ & $2.3-4.3$ \\
Serum ALP (IU/L) & $235 \pm 62$ & $132-398$ \\
Urinary NTX (nmol BCE/mmol Cr) & $73.3 \pm 21.2$ & $30.6-135.1$ \\
Number (\%) of women with & $23(48.9)$ & \\
prevalent vertebral fractures & & \\
Number (\%) of women with & $3(6.4)$ & \\
history of nonvertebral fractures & & \\
\hline
\end{tabular}

Abbreviations: SD, standard deviation; BMD, bone mineral density; YAM, young adult mean; ALP, alkaline phosphatase; NTX, cross linked N-terminal telopeptides of type I collagen; $\mathrm{BCE}$, bone collagen equivalent; $\mathrm{Cr}$, creatinine.
NTX was $73.3 \mathrm{nmol} \mathrm{BCE} / \mathrm{mmol} \mathrm{Cr}$, indicating high turnover osteoporosis (normal range 9.3-54.3 $\mathrm{nM} \mathrm{BCE} / \mathrm{mM} \mathrm{Cr}$ ). ${ }^{16}$

\section{Changes in biochemical markers and lumbar spine BMD}

Figure 1 shows the changes in the biochemical markers. Urinary NTX levels decreased to the normal range (9.3-54.3 nmol BCE/mmol Cr) ${ }^{16}$ after three months of treatment, and serum ALP levels decreased but remained within the normal range (135-310 IU/L) during the seven-year period. The mean change rate of the urinary NTX levels after three months of treatment was $-48.2 \%$. The mean change in serum ALP levels after two years of treatment was $-24.1 \%$, and this decrease continued gradually over the course of the seven-year treatment period $(-23.9 \%$ at three years, $-19.3 \%$ at five years and $-15.7 \%$ at seven years). Serum calcium levels decreased after three years of treatment, and this effect was maintained. The mean change in serum calcium level after three months was $-2.0 \%$. However, the changes in the serum phosphorus levels were not significant during the course of the seven-year treatment period.

Figure 2 shows that the lumbar spine BMD continued to increase for seven years. Increase in lumbar BMD after three, five, and seven years of treatment was $+6.1 \%,+9.4 \%$, and $+12.8 \%$, respectively.

\section{Adverse events}

No serious adverse events, including ONJ, atypical femoral diaphysis fractures, or atrial fibrillation were observed.

\section{Incident fractures}

Osteoporotic fractures including vertebral, hip, wrist, and proximal humeral fractures were assessed. During the seven-year treatment period, two patients experienced vertebral fractures and two patients experienced wrist fractures. The incidence of osteoporotic fractures was $8.6 \%$ and that of vertebral fractures was $4.3 \%$.

\section{Discussion}

To our knowledge, this is the first report showing the outcome of ALN treatment for seven years in Japanese patients with osteoporosis. ALN successfully suppressed bone turnover and increased lumbar spine BMD compared with baseline values over the seven-year treatment period without causing any severe adverse events in postmenopausal Japanese women with osteoporosis. Of importance is the effect of ALN treatment for seven years on lumbar spine BMD and bone turnover, the necessity of long-term ALN treatment, and the 

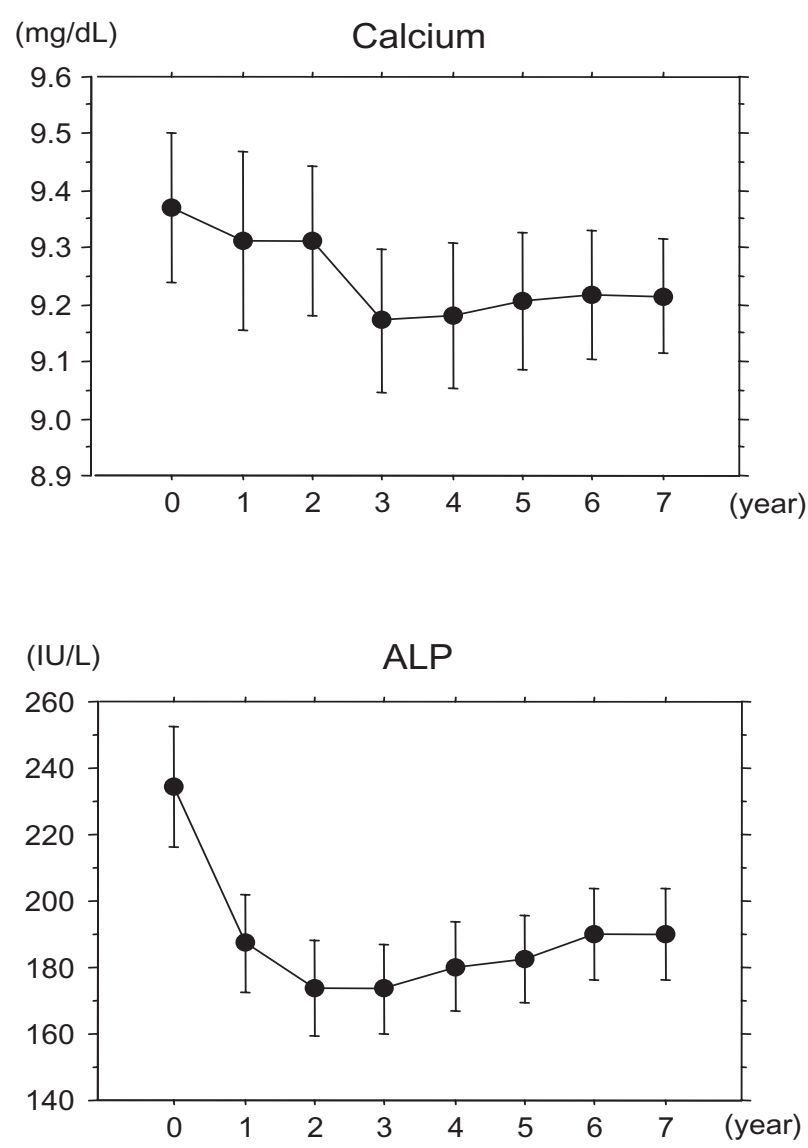

(mg/dL) Phosphorus

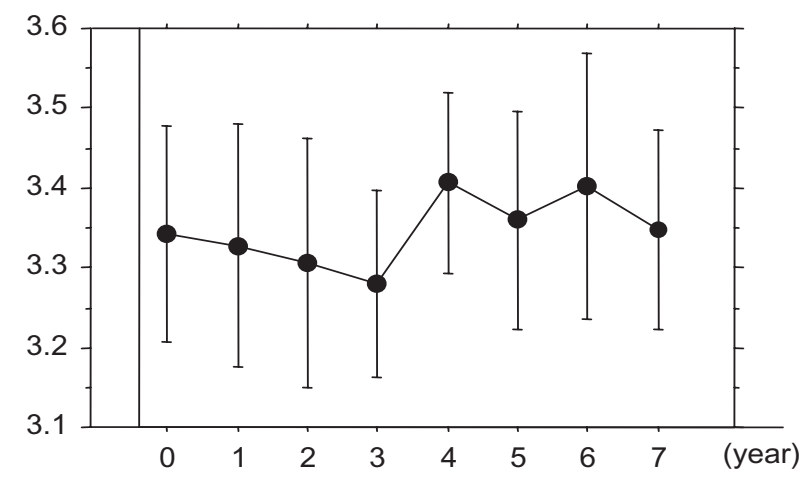

Figure I Changes in biochemical markers.

Data were expressed as the mean $\pm 95 \%$ confidence interval $(\mathrm{Cl})$ for calcium, phosphorus, and ALP and the median $\pm 95 \% \mathrm{Cl}$ for urinary NTX. One-way ANOVA with repeated measurements showed that changes in calcium and ALP, but not those in phosphorus, were significant $(P<0.000 \mathrm{I}$ and $P=0.0036$, respectively). Dashed lines are the upper (9.3 nM BCE/mM Cr) and lower (54.3 nM BCE/mM Cr) limits of urinary NTX levels. The normal range of serum ALP was I35-3।0 IU/L.

Abbreviations: ALP, alkaline phosphatase; NTX, cross-linked N-terminal telopeptides of type I collagen.

incidence of adverse events including ONJ, atypical femoral diaphysis fractures, and atrial fibrillation.

Studies have shown that ALN (5 mg daily or $35 \mathrm{mg}$ weekly) decreases urinary NTX ( $-45 \%$ at three months) and serum ALP (about $-20 \%$ at three years), and increases in lumbar BMD $(+9.2 \%$ at three years) in postmenopausal Japanese women with osteoporosis. ${ }^{6,8}$ Our previous study showed that ALN treatment for five years sustained metacarpal BMD following reductions in urinary levels of NTX $(-43.6 \%$ at three months) and serum levels of ALP ( $-18.0 \%$ at five years). In the present study, ALN treatment increased lumbar spine BMD $(6.1 \%$ at three years) subsequent to reductions in urinary levels of NTX ( $-48.2 \%$ at three months) and serum levels of ALP (-24.1\% and $-19.3 \%$ at three and five years, respectively). The results of the present study are comparable with those of previous studies.

Urinary NTX levels were reduced to within the normal range for Japanese women after three months of treatment, while serum ALP levels remained within the normal range throughout the seven-year treatment period. Measurement of urinary NTX levels was permitted only twice (just before and within six months after the start of medication) in Japan for medical insurance reason. Thus, we evaluated urinary NTX only at three months after the start of treatment, because a urinary NTX measurement performed at this time provides important information and is sufficient to monitor the effects of treatment for osteoporosis. ${ }^{15}$ We failed to show urinary NTX data after seven years of ALN treatment. However, we evaluated serum bone-specific ALP levels (normal range for Japanese women 7.9-29.0 U/L) ${ }^{16}$ after seven years of treatment. Serum levels of bone-specific ALP (mean \pm SD) at seven years was $10.0 \pm 2.0 \mathrm{U} / \mathrm{L}$, suggesting a sustained effect of ALN treatment on bone turnover for seven years.

How long postmenopausal women with osteoporosis can continue ALN treatment is debatable. A survey of the effects of treatment discontinuation would provide valuable information on this issue. Several reports have demonstrated that discontinuation of ALN affects BMD and/or levels of bone resorption markers at 1-2 years of discontinuation after 1-2 years of treatment, two years of discontinuation after 


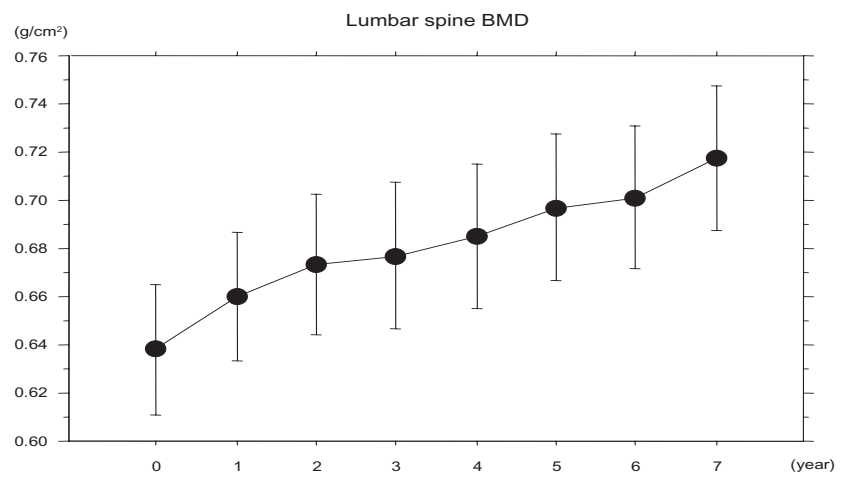

Figure 2 Changes in lumbar spine BMD.

Data were expressed as the mean $\pm 95 \%$ confidence interval $(\mathrm{Cl})$. One-way ANOVA with repeated measurements showed that changes in lumbar spine BMD were significant $(P<0.000 \mathrm{I})$

Abbreviation: BMD, bone mineral density

two years of treatment, and 3-7 years of discontinuation after 2-6 years of treatment all decreased the BMD toward the baseline level. ${ }^{17-19}$ Discontinuation of ALN treatment for five years after five years of treatment resulted in a gradual loss of its effects, as measured using BMD and biochemical markers of bone turnover, ${ }^{4}$ and increased the risk of clinical vertebral fractures, compared with continuation of ALN treatment. ${ }^{20} \mathrm{~A}$ recent study also showed that among women without any vertebral fractures after five years of ALN treatment, continuation of ALN treatment for another five years reduced the risk of nonvertebral fractures in women with a femoral neck BMD T-score $\leq-2.5$ at five years, but not in women with a T-score $>-2$, compared with the discontinuation of ALN treatment for five years after five years of treatment. ${ }^{21}$ These results suggest that continuous ALN treatment for 10 years is optimal for selected patients.

If patients are not considered to have a high risk of fractures, the discontinuation of ALN treatment could probably be considered after treatment for five years, with subsequent monitoring of BMD and bone turnover markers. The most important risk factor for fractures is considered to be BMD. ${ }^{21}$ Other risk factors are low-trauma fractures after the age of 40 years, a maternal history of osteoporotic fractures after the age of 65 years, a thin body build, prolonged amenorrhea, early menopause, chronic corticosteroid use ( $>$ six months), and diseases predisposing an individual to osteoporosis. ${ }^{22}$ In the present study, treatment was continued in all patients because they had BMD below $70 \%$ of the YAM or at least one of the aforementioned risk factors, as evaluated at the lumbar spine after five years of ALN treatment.

ONJ, atypical femoral diaphysis fractures, and atrial fibrillation have been reported as severe adverse events, ${ }^{10-12}$ although whether these events are significantly related to ALN treatment remains uncertain. The efficacy and safety of ALN for 10 years has been established in postmenopausal Western women with osteoporosis. ${ }^{4}$ However, adherence (persistence and compliance) of patients to treatment with oral bisphosphonates is very poor. ${ }^{23}$ Because patients who withdrew from treatment were not followed up, the long-term safety of ALN remains uncertain in this patient group.

Once-yearly intravenous zoledronate has been reported to prevent morphometric vertebral, nonvertebral, and hip fractures in postmenopausal women with osteoporosis effectively (relative risk $0.30,0.75$, and 0.59 , respectively) ${ }^{24}$ and reduce mortality in patients who suffer a low-trauma hip fracture..$^{25}$ Because adherence to treatment with once-yearly intravenous zoledronate may be better than that with oral alendronate in clinical practice, zoledronate could be more useful in the prevention of osteoporotic fractures and reduce medical expenses related to treatment of hip fractures.

The incidence of vertebral fractures was $4.3 \%$ in the present study. A RCT of the two-year minodronate study showed that the incidence of vertebral and nonvertebral fractures in postmenopausal Japanese controls with established osteoporosis was $21.3 \%$ and $3.5 \%$, respectively. ${ }^{26}$ However, a three-year RCT of ALN showed that the incidence of vertebral fractures was $2 \%$ for the ALN group and $8 \%$ for the active control (alfacalcidol) group in postmenopausal Japanese women with osteoporosis. ${ }^{6}$ One possible explanation for the higher incidence of vertebral fractures in the present study might be that there was a higher proportion of patients who had prevalent vertebral fractures in terms of higher risk of incident fractures at baseline as well as a comparatively long duration of observation. Another possibility might be the existence of subclinical osteomalacia on a background of significantly decreased serum calcium levels because of lack of calcium and vitamin D supplementation.

The present study confirmed the effect of ALN on lumbar spine BMD and bone turnover in postmenopausal Japanese women with osteoporosis who continued treatment for seven years. However, this study has notable limitations. First, it was a retrospective cohort study with a small sample size. Second, the subjects did not receive either elementary calcium or natural vitamin D supplementation. Natural vitamin D supplementation is not routinely given in Japan. This makes it difficult to compare our study with those of others, as most other studies have included postmenopausal women with osteoporosis taking calcium and vitamin D supplements. Moreover, performing a seven-year RCT with a sufficient number of subjects is difficult, but prospective studies with 
a large number of subjects are needed to establish the longterm efficacy and safety of ALN treatment with calcium and vitamin D supplementation.

In conclusion, the present retrospective study showed that ALN successfully suppressed bone turnover and increased lumbar spine BMD, compared with baseline values, over the course of a seven-year treatment period without causing any severe adverse events in postmenopausal Japanese women with osteoporosis.

\section{Disclosures}

The authors report no funding sources or conflict of interest in this work.

\section{References}

1. Black DM, Cummings SR, Karpf DB, et al. Randomised trial of effect of alendronate on risk of fracture in women with existing vertebral fractures. Lancet. 1996;348:1535-1541.

2. Cummings SR, Black DM, Thompson DE, et al. Effect of alendronate on risk of fracture in women with low bone density but without vertebral fractures: Results from the Fracture Intervention Trial. JAMA. 1998;280:2077-2082.

3. Wells GA, Cranney A, Peterson J, et al. Alendronate for the primary and secondary prevention of osteoporotic fractures in postmenopausal women. Cochrane Database Syst Rev. 2008;23(1):CD001155.

4. Bone HG, Hosking D, Devogelaer JP, et al. Alendronate Phase III Osteoporosis Treatment Study Group. Ten years' experience with alendronate for osteoporosis in postmenopausal women. NEngl J Med. 2004;350:1189-1199.

5. Kushida K, Shiraki M, Nakamura T, et al. The efficacy of alendronate in reducing the risk for vertebral fracture in Japanese patients with osteoporosis: A randomized, double-blind, active-controlled, double-dummy trial. Curr Ther Res. 2002;63:9:606-620.

6. Kushida K, Shiraki M, Nakamura T, et al. Alendronate reduced vertebral fracture risk in postmenopausal Japanese women with osteoporosis: A 3-year follow-up study. J Bone Miner Metab. 2004;22:462-468.

7. Shiraki M, Kushida K, Fukunaga M, et al. A double-masked multicenter comparative study between alendronate and alfacalcidol in Japanese patients with osteoporosis. Osteoporos Int. 1999;10:183-192.

8. Uchida S, Taniguchi T, Shimizu T, et al. Therapeutic effects of alendronate $35 \mathrm{mg}$ once weekly and $5 \mathrm{mg}$ once daily in Japanese patients with osteoporosis: A double-blind, randomized study. J Bone Miner Metab. 2005;23:382-388.

9. Iwamoto J, Miyata A, Sato Y, et al. Five-year alendronate treatment outcome in older postmenopausal Japanese women with osteoporosis or osteopenia and clinical risk factors for fractures. Ther Clin Risk Manag. 2009;5:773-779.

10. Ruggiero SL, Dodson TB, Assael LA, et al. American Association of Oral and Maxillofacial Surgeons position paper on bisphosphonate-related osteonecrosis of the jaws - 2009. Update. J Oral Maxillofac Surg. 2009;67:2-12.

Therapeutics and Clinical Risk Management

\section{Publish your work in this journal}

Therapeutics and Clinical Risk Management is an international, peerreviewed journal of clinical therapeutics and risk management, focusing on concise rapid reporting of clinical studies in all therapeutic areas, outcomes, safety, and programs for the effective, safe, and sustained use of medicines. This journal is indexed on PubMed Central, CAS,
11. Lenart BA, Lorich DG, Lane JM. Atypical fractures of the femoral diaphysis in postmenopausal women taking alendronate. $N$ Engl J Med. 2008;358:1304-1306.

12. Heckbert SR, Li G, Cummings SR, et al. Use of alendronate and risk of incident atrial fibrillation in women. Arch Intern Med. 2008;168: 826-831.

13. Orimo H, Sugioka Y, Fukunaga M, et al. Diagnostic criteria of primary osteoporosis. J Bone Miner Metab. 1998;16:139-150.

14. Orimo H, Hayashi Y, Fukunaga M, et al. Diagnostic criteria for primary osteoporosis: Year 2000 revision. J Bone Miner Metab. 2001;19: 331-337.

15. Iwamoto J, Takeda T, Sato Y, et al. Early changes in urinary cross-linked $\mathrm{N}$-terminal telopeptides of type I collagen level correlate with one-year response of lumbar bone mineral density to alendronate in Japanese postmenopausal women with osteoporosis. J Bone Miner Metab. 2005;23:238-242.

16. Nishizawa Y, Nakamura T, Ohta H, et al. Guidelines for the use of biochemical markers of bone turnover in osteoporosis (2004). J Bone Miner Metab. 2005;23:97-104.

17. Ravn P, Bidstrup M, Wasnich RD, et al. Alendronate and estrogen-progestin in the long-term prevention of bone loss: Four-year results from the early postmenopausal intervention cohort study. A randomized, controlled trial. Ann Intern Med. 1999;131:935-942.

18. Stock JL, Bell NH, Chesnut CH III, et al. Increments in bone mineral density of the lumbar spine and hip and suppression of bone turnover are maintained after discontinuation of alendronate in postmenopausal women. Am J Med. 1997;103:291-297.

19. Bagger YZ, Tanko LB, Alexandersen $P$, et al. Alendronate has a residual effect on bone mass in postmenopausal Danish women up to seven years after treatment withdrawal. Bone. 2003;33:301-307.

20. Black DM, Schwartz AV, Ensrud KE, et al. Effects of continuing or stopping alendronate after 5 years of treatment: The Fracture Intervention Trial Long-term Extension (FLEX): A randomized trial. JAMA. 2006;296:2927-2938.

21. Schwartz AV, Bauer DC, Cauley A, et al. Efficacy of continued alendronate for fractures in women without prevalent vertebral fracture: The FLEX trial (Abstr 1057). Presented at the 29th Annual Meeting of the American Society for Bone and Mineral Research. Honolulu, HI; September 16-20, 2007.

22. Meunier PJ, Delmas PD, Eastell R, et al. Diagnosis and management of osteoporosis in postmenopausal women: Clinical guidelines. International Committee for Osteoporosis Clinical Guidelines. Clin Ther. 1999;21:1025-1044.

23. Rabenda V, Hiligsmann M, Reginster JY. Poor adherence to oral bisphosphonate treatment and its consequences: A review of the evidence. Expert Opin Pharmacother. 2009;10:1-13.

24. Black DM, Delmas PD, Eastell R, et al. Once-yearly zoledronic acid for treatment of postmenopausal osteoporosis. $N$ Engl J Med. 2007;356:1809-1822.

25. Eriksen EF, Lyles KW, Colón-Emeric CS, et al. Antifracture efficacy and reduction of mortality in relation to timing of the first dose of zoledronic acid after hip fracture. J Bone Miner Res. 2009;24:1308-1313.

26. Matsumoto T, Hagino H, Shiraki M, et al. Effect of daily oral minodronate on vertebral fractures in Japanese postmenopausal women with established osteoporosis: A randomized placebo-controlled double-blind study. Osteoporos Int. 2009;20:1429-1437.

EMBase, Scopus and the Elsevier Bibliographic databases. The manuscript management system is completely online and includes a very quick and fair peer-review system, which is all easy to use. Visit http://www.dovepress.com/testimonials.php to read real quotes from published authors. 